\title{
Scaling relations for particles whose velocities are randomly reset
}

\author{
Yuh-Hwa Chang, Yih-Yuh Chen ${ }^{1}$ \\ Physics Department, National Taiwan University, Taipei. Taiwan, ROC
}

Received 15 July 1998; revised manuscript received 13 October 1998; accepted for publication 13 October 1998

Communicated by C.R. Doering

\begin{abstract}
While it is generally the case that the trajectory of a particle placed inside a confining potential is chaotic, under certain conditions this trajectory might become non-chaotic when its velocity is periodically reset to a randomly chosen value. We suggest a way of analyzing this phenomenon and derive several scaling relations for this situation. Physical interpretations of the derived results are also discussed. (c) 1998 Elsevier Science B.V.
\end{abstract}

PACS: $05.45 .+\mathrm{b}$

Although chaos in itself implies indeterminacy or uncertainty in the prediction of the future evolution of a dynamical system, we now have ample examples indicating that chaotic behavior might be suppressed if a certain perturbation is imposed on the otherwise chaotic system. One famous example is the work of Pecora and Carroll [1], in which it is shown that a chaotic slave system exhibits no sensitivity on initial conditions if a judiciously chosen variable of the slave is replaced by that of an identical master system. Still another example is the work of Fahy and Hamann [2], in which it is demonstrated that identical particles placed inside a confining potential might eventually converge in their orbits if the velocities of the particles are periodically reset to a common randomly chosen value. The latter had been further studied by Kaulakys and Vektaris [3], Maritan and Banavar [4], and by Chen [5]. In particular, a notable feature of Ref. [5] is the suggestion that this convergence of orbits is of a rather general nature. In view of the generality implied by this work, a more satisfactory treatment than that in Ref. [5] seems desirable. It is the purpose of this Letter to (1) present an addition to the cited references using a more coherent treatment, $(2)$ generalize the analysis to the case when the system has a memory effect, and (3) derive new scaling relations and provide a physical explanation.

One conclusion derived from our present work is that it is not essential to have an isotropic distribution in the random reset velocity for the orbits to converge. But more importantly, we would like to point out that the partial reduction in the velocity after each reset (that is, the $\alpha$ to be defined below being less than unity) mimicking the memory effect actually affects the system in a way quite different from that caused by dissipation, despite their superficial resemblance of being equally capable of reducing the original velocity. The full explanation of

${ }^{1}$ Corresponding author. 
this counter-intuitive prediction will be given below after we have worked out the corresponding mathematics to support the claim based on physical reasoning.

To begin with, we suppose that two non-interacting identical particles are put inside a confining force field and write the external potential energy as $V(\boldsymbol{x})$, with $\boldsymbol{x}$ denoting the coordinates of a particle. For definiteness, we will consider $[2,5]$

$$
V(x, y)=\sin (2 \pi x) / 2 \pi x+\sin (2 \pi y) / 2 \pi y+\left(x^{2}+y^{2}\right)^{s / 2} / 16 \pi
$$

and use $s=4$ as illustration, though all the predictions in this Letter have also been verified for $s=6$ as well. The equation of motion for either particle is assumed to be

$$
\frac{\mathrm{d} \boldsymbol{x}}{\mathrm{d} t}=\boldsymbol{v}, \quad m \frac{\mathrm{d} \boldsymbol{v}}{\mathrm{d} t}=-\frac{\partial V}{\partial \boldsymbol{x}}-\gamma \boldsymbol{v},
$$

where $m$ is the mass of the particles, $v$ the velocity, and we have included a damping factor $\gamma \geqslant 0$ to take into account possible dissipative effects. The system is allowed to freely evolve for a time $\tau$ before the particle velocities are reset to a new value $\boldsymbol{v}_{\text {new }}$, which takes the following form,

$$
\boldsymbol{v}_{\text {new }}=\alpha v_{\text {old }}+\beta v_{\text {random }} \text {. }
$$

Here, the parameter $\alpha$ is meant to simulate a certain memory effect $(0 \leqslant \alpha \leqslant 1)$, and $\boldsymbol{v}_{\text {random }}$ is a velocity randomly selected from a prescribed normalized distribution (the average of $v_{\text {random }}^{2}$ is some convenient constant). Also, $\beta>0$ is a tunable parameter signifying the magnitude of the random velocity.

Because it has been reported that for the non-dissipative case $(\gamma=0)$ the particles will eventually converge in orbits for all periodic reset times $\tau$ less than some threshold $\tau_{c}$, a natural starting point for the understanding of this behavior is the investigation of the linear stability of the system when $\tau$ is small. This can be facilitated by computing the small deviation $\delta \boldsymbol{x}$ (in position) and $\delta \boldsymbol{v}$ (in velocity) of two neighboring trajectories. Using a subscript " 0 " to denote the initial value of a variable, and defining

$$
H_{0} \equiv\left(\begin{array}{cc}
I & 0 \\
0 & \alpha I
\end{array}\right)+\tau\left(\begin{array}{cc}
0 & I \\
-\frac{\alpha}{m} \frac{\partial^{2} V}{\partial \boldsymbol{x}_{0}^{2}} & -\frac{\alpha \gamma}{m} I
\end{array}\right)-\frac{\tau^{2}}{2 m}\left(\begin{array}{cc}
\frac{\partial^{2} V}{\partial \boldsymbol{x}_{0}^{2}} & \gamma I \\
\alpha \frac{\partial^{3} V}{\partial \boldsymbol{x}_{0}^{3}} \boldsymbol{v}_{0}-\frac{\alpha \gamma}{m} \frac{\partial^{2} V}{\partial \boldsymbol{x}_{0}^{2}} & \alpha \frac{\partial^{2} V}{\partial \boldsymbol{x}_{0}^{2}}-\frac{\alpha \gamma^{2}}{m} I
\end{array}\right),
$$

it is straightforward to show that $(\delta \boldsymbol{x}, \delta \boldsymbol{v})^{T}=H_{0}\left(\delta \boldsymbol{x}_{0}, \delta \boldsymbol{v}_{0}\right)^{T}$, where $I$ is the $D \times D$ identity matrix $(D=$ spatial dimension), provided we keep only up to the second order of $\tau$. The accumulated effect of the matrices $H_{0}, H_{1}, \ldots$ (at different times) can be computed with the help of the Baker-Campbell-Hausdorff formula

$$
\mathrm{e}^{A} \mathrm{e}^{B} \approx \exp \left(A+B+\frac{1}{2}[A, B]+\frac{1}{12}([A,[A, B]]+[B,[B, A]])\right)
$$

once we have recast Eq. (3) into exponential form. For instance, for the case $\alpha=\gamma=0$ studied in detail in Ref. [5] the above reduces to $\delta \boldsymbol{x} \approx h_{0} \delta \boldsymbol{x}_{0}$ for a matrix $h_{0}$ defincd as

$$
h_{0}=\exp \left(-\frac{\tau^{2}}{2 m} \frac{\partial^{2} V}{\partial x_{0}^{2}}\right)
$$

(correct to second order in $\tau$ ) so that after time $t=n \tau$ we have

$$
\delta \boldsymbol{x}_{n} \approx h_{n-1} h_{n-2} \ldots h_{0} \delta \boldsymbol{x}_{0} \approx \exp \left[-\frac{\tau^{2}}{2 m}\left(\frac{\partial^{2} V}{\partial \boldsymbol{x}_{n-1}^{2}}+\ldots+\frac{\partial^{2} V}{\partial \boldsymbol{x}_{0}^{2}}\right)\right] \delta \boldsymbol{x}_{0}=\exp \left(-\frac{n \tau^{2}}{2 m}\left\langle\frac{\partial^{2} V}{\partial \boldsymbol{x}^{2}}\right\rangle\right) \delta \boldsymbol{x}_{0},
$$

where the angular brackets stand for the average value of a quantity. Employing the argument of Ref. [5] one can show that $L \equiv\left\langle\partial^{2} V / \partial \boldsymbol{x}^{2}\right\rangle / 2 m$ is non-negative for a confining potential of reflection symmetry, thus 
implying that chaos is suppressed for all small enough $\tau$. In addition, the above derivation involves no explicit dependence on the velocity, suggesting that even an anisotropic velocity distribution still gives rise to the same conclusion as long as its mean is zero (so that the particles will not drift away to infinity). We have verified this prediction as well.

As another example, we can consider the case $\alpha \equiv 1-\epsilon, \epsilon$ being a small positive number. After some tedious algebra (due to the presence of the commutators), onc arrives at

$$
\begin{aligned}
& \left(\begin{array}{l}
\delta \boldsymbol{x}_{n} \\
\delta v_{n}
\end{array}\right) \approx\left(\exp n\left(\begin{array}{cc}
-\frac{1}{6}-\epsilon \tau^{2} L & \tau\left(1+\frac{1}{2} \epsilon\right) I-\left(\epsilon \gamma \tau^{2} / 12 m\right) I \\
-\tau\left(1-\frac{1}{2} \epsilon\right) L+\epsilon \gamma \tau^{2} L / 12 m & -(\epsilon+\gamma \tau / m) I+\frac{1}{6} \epsilon \tau^{2} L
\end{array}\right)\right)\left(\begin{array}{l}
\delta \boldsymbol{x}_{0} \\
\delta \boldsymbol{v}_{0}
\end{array}\right) \\
& \equiv A^{n}\left(\begin{array}{l}
\delta x_{0} \\
\delta v_{0}
\end{array}\right),
\end{aligned}
$$

which is correct only to first order in $\epsilon$, and where terms such as

$$
\left(1-\frac{1}{n}\right) \frac{\partial^{2} V}{\partial \boldsymbol{x}_{n-1}^{2}}+\left(1-\frac{3}{n}\right) \frac{\partial^{2} V}{\partial \boldsymbol{x}_{n-2}^{2}}+\ldots-\left(1-\frac{3}{n}\right) \frac{\partial^{2} V}{\partial \boldsymbol{x}_{1}^{2}}-\left(1-\frac{1}{n}\right) \frac{\partial^{2} V}{\partial \boldsymbol{x}_{0}^{2}}
$$

and the like have been summed to zero on account of the randomness of the trajectory. (We have also numerically checked this assumption.)

The eigenvalue of $A$ is

$$
\lambda=\exp \left\{\frac{1}{2}\left[-(\epsilon+\gamma \tau / m) \pm \sqrt{(\epsilon+\gamma \tau / m)^{2}-4 l \tau^{2}}\right]\right\}
$$

where $l$ is the (necessarily non-negative) eigenvalue of $L$ defined before. Since the above was obtained under the assumption of $\tau$ being small, we must not allow either $\gamma / \mathrm{m}$ or $l$ to be unreasonably large if we are to have self-consistency. In addition, we can not directly set the real part of $\lambda$ to zero to derive an expression for the threshold time $\tau_{c}$. However, Ref. [5] suggests that one can still extract certain information about $\tau_{c}$ from the fact that the two terms in the radical must be of the same magnitude when $\tau=\tau_{c}$. Thus, we expect

$$
\epsilon+\frac{\gamma \tau_{c}}{m} \propto \sqrt{l} \tau_{c}
$$

To proceed further, however, we need a relation between the average velocity $\boldsymbol{\nu}_{\text {ave }}$ and the parameter $\epsilon$ (or $\alpha$ ). This can be done as follows.

Because the particle retains some memory of its previous velocity after each reset through the parameter $\alpha \neq 0$, one cannot use the analogy from Brownian motion to find a relation between $l$ and the external

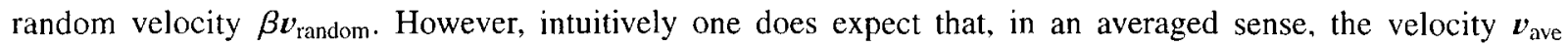
must somehow recover itself in between two consecutive velocity resets. Thus, to have self-consistency, we should have

$$
\left[\left(1-\frac{\gamma \tau}{m}\right)\left(\alpha v_{\text {ave }}+\beta v_{\text {random }}\right)-\frac{\tau}{m} \frac{\partial V}{\partial \boldsymbol{x}}\right]^{2} \approx\left(v_{\text {ave }}\right)^{2},
$$

where only the first-order term in the small free evolution time $\tau$ is retained. This gives

$$
\left\|\boldsymbol{v}_{\text {ave }}\right\| \propto \frac{\beta}{\sqrt{1+2 \gamma \tau / m-\alpha^{2}}},
$$

where use has been made of the fact that $\boldsymbol{v}_{\text {ave }} \cdot \boldsymbol{v}_{\text {random }}=0, \boldsymbol{v}_{\text {randon }} \cdot \partial V / \partial \boldsymbol{x}=\boldsymbol{v}_{\text {ave }} \cdot \partial V / \partial \boldsymbol{x}=0$ after averaging over phase space. Eq. (12) is well verified in Fig. I for the simpler case when $\gamma=0$. (In this and all the simulations reported below, we have fixed the distribution for $\boldsymbol{v}_{\text {random }}$ so that it is isotropic and has a magnitude uniformly distributed in the interval $[0,250]$.) 


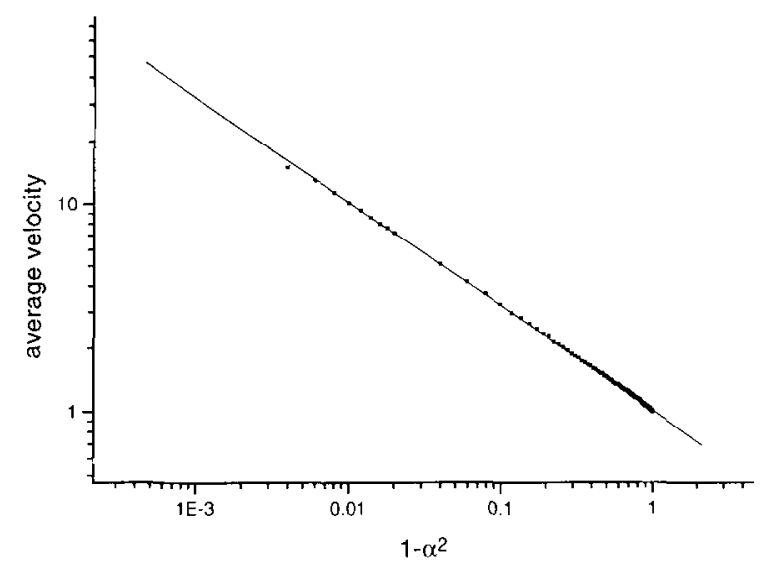

Fig. 1. Log-log plot of the average velocity $\| v^{\prime}$ ave $\|$ versus $1-\alpha^{2}$. The slope is -0.50 , in agreement with the theoretical prediction of $-1 / 2$.

Setting the average kinetic energy $m v_{\text {avc }}^{2} / 2$ of a particle to be approximately equal to its average potential energy, we may easily derive from Eq. (10) the following relation,

$$
\tau_{c} \propto m^{1 / s}\left(\epsilon+\frac{\gamma \tau_{c}}{m}\right)\left(\frac{\beta}{\sqrt{1+2 \gamma \tau_{c} / m-(1-\epsilon)^{2}}}\right)^{-1+2 / s},
$$

provided that, on the average, $V \propto r^{s}$ is a good approximation in the calculation. For the special case of $\gamma=0$ we have

$$
\tau_{c} \times m^{3 / s} \epsilon\left(2 \epsilon-\epsilon^{2}\right)^{0.5-1 / s} \beta^{-1+2 / s} \quad(\gamma=0)
$$

which is verified in Fig. 2 for $s=4$. The physically more realistic case of $\alpha=1$ reduces Eq. (13) to

$$
\tau_{c} \propto m^{(3 s-4) /(s-2)} \gamma^{(-3 s+2) /(s-2)} \beta^{2} \quad(\alpha=1),
$$

which is shown in Fig. 3. In contrast to the previous figures, this time our agreement is only modest (with a ten percent discrepancy). Our past experience with numerical simulations suggests that this discrepancy is probably caused by numerical errors, because it usually takes a much longer time to observe convergence of the orbits when $\alpha=1$, and any premature ending of the simulation due to an improperly chosen criterion for convergence is bound to widen the discrepancy.

We now discuss the physics behind the derivations. Again, we will take $s=4$ as illustration. The first thing we can notice is that Eq. (9) implies that orbits are expected to converge for all small enough $\tau$ when $\gamma=0$. Though we mentioned before that this, or more specifically, Eq. (14), is borne out by our numerical simulations, the physics is not completely spelled out if we restrict ourselves entirely to this case. In fact, the orbits can also converge for all large enough $\tau$ when $y$ is nonvanishing. The reason behind this scenario is simple: $\gamma$ causes dissipation, and whenever we are willing to wait for a long enough time the particle will eventually settle down to some local minimum of the potential. The periodic kicks after each long waiting time $\tau$ simply serve to "anneal" the particles so that they will have a probability to chance into the same local potential minimum. In other words, as long as $\gamma \neq 0$ there should exist a window such that orbital convergence is impossible for any $\tau$ inside $\mathrm{it}^{2}$. For instance, with $s=4, m=10, \beta=0.4$ and $\gamma=5$ the window has been determined to be $[0.014,3.2]$. The only question is: How do the boundaries of this window depend on the system parameters?

\footnotetext{
2 The existence of a lower bound to this window was already observed in Ref. [6]
} 

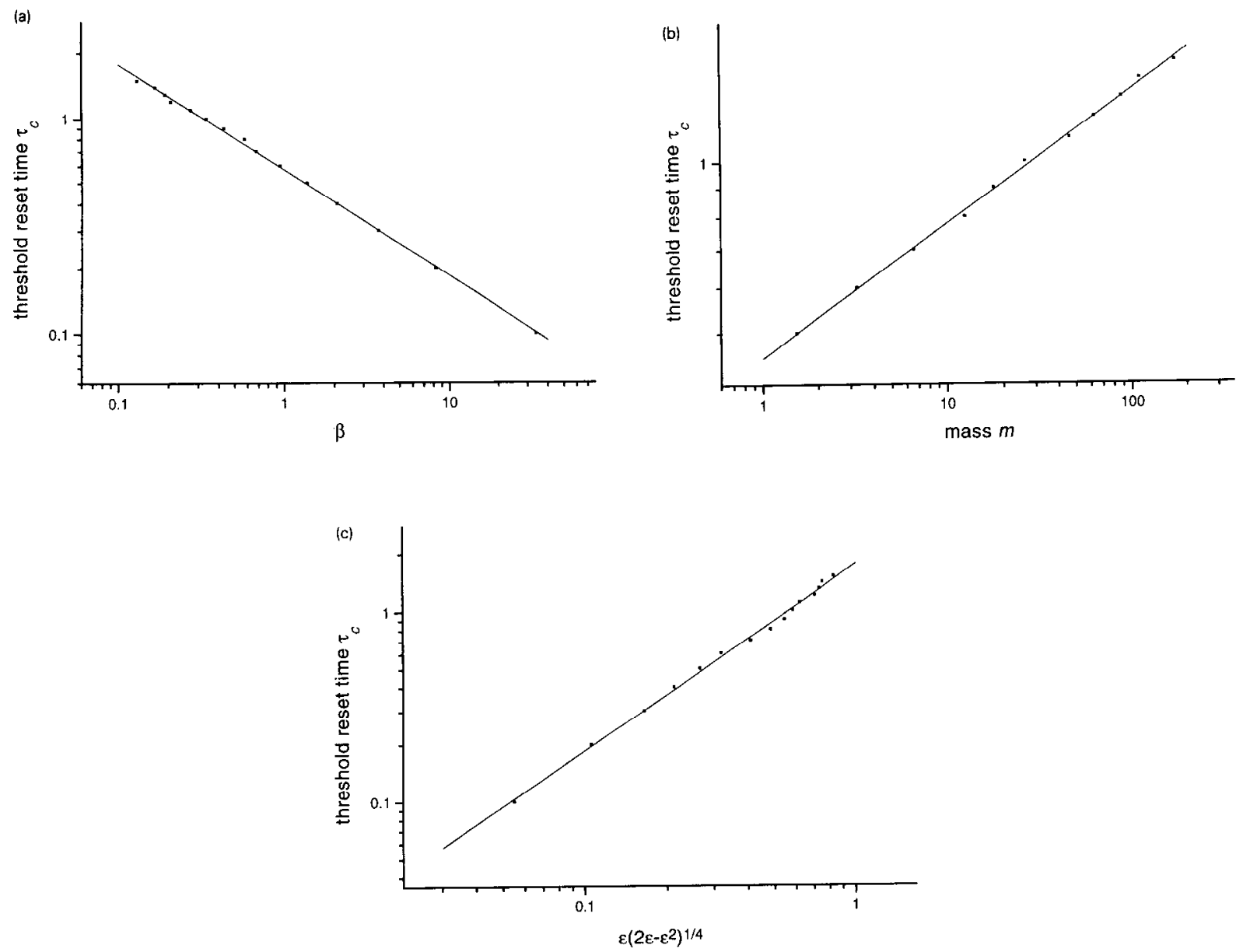

Fig. 2. $\tau_{c}$ versus (a) $\beta$ when $m=20, \alpha=0.5$, (b) $m$ when $\beta=0.4, \alpha=0.5$, and (c) $\epsilon\left(2 \epsilon-\epsilon^{2}\right)^{1 / 4}$ when $m=10, \beta=0.4$. ( $\gamma$ is set to zero in all the cases.) The slopes are respectively (a) -0.49 , (b) 0.24 , and (c) 0.97 , comparing favorably with the theoretical values of $-1 / 2,1 / 4$, and 1 .

In this regard, Eq. (14) provides us with a partial answer for the lower boundary, whereas Eq. (15) predicts that for the upper boundary. We can see why Eq. (15) refers to the upper boundary in two ways: (1) it cannot be describing the lower boundary because Eq. (14) asserts that the lower boundary tends to zero as $\alpha \longrightarrow 1^{-}$; (2) the aforementioned annealing argument suggests that the upper boundary should approach zero when we have a large $\gamma$, and this is exactly what Eq. (15) implies.

Yet another way of looking at the physics underlying this problem is to identify two different mechanisms that are capable of making the orbits converge. First, we note that the time scale $\tau_{1}$ for the small deviation $\|\delta x\|$ to be compressed by the curvature of the potential has an order of magnitude satisfying

$$
\tau_{1}^{-2}=\mathrm{O}\left(\frac{1}{m} \frac{\partial^{2} V}{\partial x^{2}}\right)=O(l)
$$

If the waiting time $\tau$ is too large compared to $\tau_{1}$ when no dissipation is present, the two particles will begin to separate because they have passed the points of closest approach. Thus, the criterion for the lower threshold of $\tau_{c}$ is simply $\tau_{c} \sim \tau_{1}$. This argument immediately leads one to Eq. (14) if we adopt the way of estimating $l$ described above. But if $\alpha=1$ and $\gamma$ is large, then the effect of dissipation is to break apart the two particles 

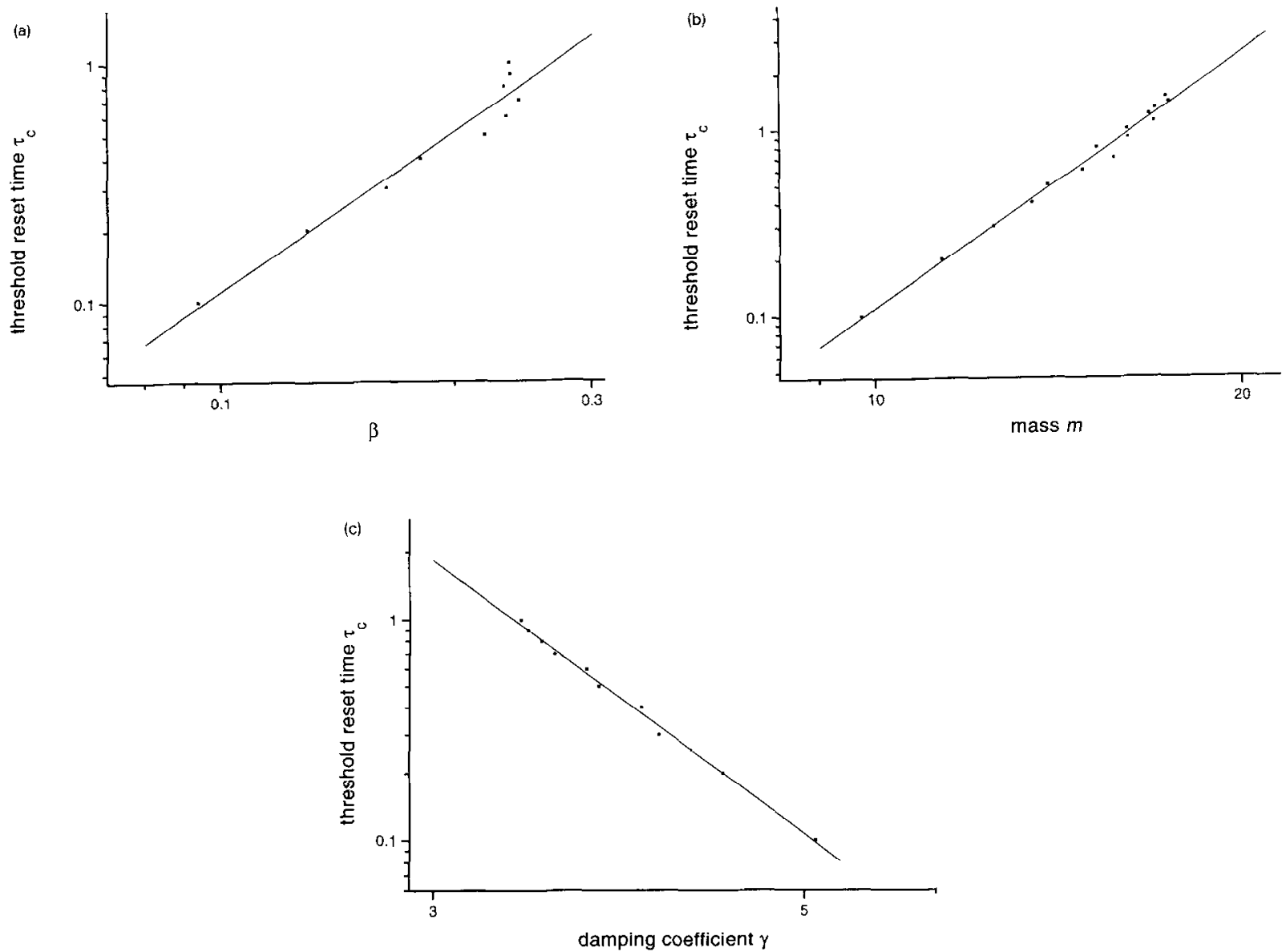

Fig. 3. $\tau_{c}$ versus (a) $\beta$ when $m=10, \gamma=5$, (b) $m$ when $\beta=0.1, \gamma=5$, and (c) $\gamma$ when $m=10 . \beta=0$. 1 . (All cases plotted here are for $\alpha=1$.) The slopes are respectively (a) 2.2 , (b) 4.5 , and (c) -5.6 , agreeing modestly (about ten percent in error) with the theoretical values of 2,4 , and -5 .

and let them each dissipate their entire energy and come to a potential minimum before the annealing process described above takes over and eventually brings the orbits together (by chance). This mechanism is effective only if the dissipation time scale

$$
\tau_{2} \equiv m / \gamma
$$

is greater than $\tau_{1}$. Due to the nature of this mechanism, we see that the threshold time $\tau_{c}$ chus obtained clearly corresponds to the upper boundary of the window of inoperative $\tau$. Again, one easily verifies that $\tau_{1} \sim \tau_{2}$ indeed gives the correct scaling predicted by Eq. (15).

In conclusion, a more systematic treatment of the stability of particles placed inside a confining potential is presented when their velocities are subject to periodic resets. We demonstrated how scaling relations for the parameter dependence of the (lower and/or upper) threshold reset time $\tau_{c}$ can be derived using this formalism. The scaling relations seem to admit simple physical interpretations when appropriate limits are taken. Though far from being complete, the arguments presented might nevertheless serve as a guide for further study and the eventual formulation of a more rigorous theory. 
This work was supported by the National Science Council of the Republic of China under grant number NSC87-2112-M-002-018.

\section{References}

(1) L.M. Pecora, T.L. Carroll. Phys. Rev. Lett. 64 (1990) 821

[2] S. Fahy, D.R. Hamann, Phys. Rev. Lett. 69 (1992) 761.

13] B. Kaulakys, G. Vektaris, Phys. Rev. E 52 (1995) 2091.

14| A. Maritan, J. R. Banavar, Phys. Rev. Lett. 72 (1994) 1451; A.S. Pikovsky, Phys. Rev. Lett. 73 (1994) 2931;

A. Maritan, J.R. Banavar, Phys. Rev. Lett. 73 (1994) 2932.

|5| Y.-Y. Chen. Phys. Rev. Lett. 77 (1996) 4318.

16| H.-T. Chen, Synchronizing Chaotic Systems Using a Random Driving, Master Thesis. National Taiwan University (1996). 